\title{
CrimRxiv
}

\section{Factors that predict the referral of adult Modern Day Slavery cases to the UK's National Referral Mechanism}

Freya O'Brien, Sasha Palmer, Victoria Blinkhorn

Published on: Feb 26, 2022

DOI: $10.21428 / \mathrm{cb} 6 \mathrm{ab} 371.273631 \mathrm{eb}$

License: Creative Commons Attribution 4.0 International License (CC-BY 4.0). 
